\title{
Naturally Acquired Antibodies Against the Major Merozoite Surface Coat Protein (MSP-1) of Plasmodium falciparum Acquired by Residents in an Endemic Area of Colombia
}

\author{
Zilka I Terrientes, Kenton Kramer*, Myriam A Herrera**, Sandra P Chang*
}

Department of Microbiology and Center for Research and Diagnostic of Parasitic Diseases, Faculty of Medicine, University of Panama, Panama *Department of Tropical Medicine and Medical Microbiology, John Burns, School of Medicine, University of Hawaii, Honolulu, Hawaii, USA ** Department of clinical laboratory, School of Health,

Universidad del Valle, Cali, Colombia

A preliminary baseline epidemiological malaria survey was conducted in the village of Punta Soldado, Colombia. Parasite prevalence and density as well as serological data were obtained from 151 asymptomatic children and adults. Fifty individuals were infected with Plasmodium falciparum. The mean parasite density was 184 parasites $/ \mathrm{mm}^{3}$. Greater than $90 \%$ of the sample population were P. falciparum antibody positive as detected by the indirect immunofluorescent antibody test (IFAT). The enzyme-linked immunosorbent assay (ELISA) was used to detect antibodies against the major merozoite surface protein $(M S P-1)$ of $\mathrm{P}$. falciparum. In this population, anti-MSP-I antibody concentration is acquired in an age dependent manner with equal immunogenicity to both the $N$ - and $C$-terminal regions of the molecule. Infection at the time of sampling was associated with a higher anti-MSP-1 antibody concentration than that found in non-infected individuals. Further studies are planned to assess the role of immune and non-immune factors in limiting the number of cases of severe malaria seen in this population.

Key words: Plasmodium - immunity - antibody - asexual stages

Malaria is a significant cause of morbidity and mortality in tropical and subtropical regions of the world. In endemic areas, malaria mortality is restricted mainly to young children. Immunity to the disease, but not to infection, develops and is maintained through repeated infections. Human malaria studies have demonstrated that the development of immunity requires repeated infections with prolonged exposure of susceptible hosts to many antigenically-distinct parasite strains, or repeated exposure to poorly immunogenic epitopes involved in protection (McGregor 1988). Taylor (1989) hypothesized that repeated exposure to the parasite would result in (1) increased antibody titers, (2) recognition of antigens not previously recognized (i.e. minor antigenic determinants or antigenic variation) and/or increased antibody quality (i.e., affinity, avidity, isotype, etc.). Numerous seroepidemiological studies have been conducted aimed at explaining the role of the humoral immune

This study received financial support from Fulbright Research Grant and AID Collaborative Agreement No. HRN-6001-A-002020-00. response in naturally acquired immunity to malaria. Although there is an association between the number of malaria infections and antibody titer, it has been difficult to consistently demonstrate a relationship between anti-malarial antibody titers and protection from infection or disease. This paradox maybe due to the fact that investigators have been examining different malarial antigens under different endemic conditions.

Due to the increasing complexity and difficulty of malaria control, vaccination against malaria has taken on increased importance. One of the primary candidate antigens for a vaccine against $P$. falciparum is MSP-1, also known as MSA-1, gp195, p190 and PMMSA (Hall et al. 1984, Perrin et al. 1984, Cheung et al. 1986, Siddiqui et al. 1987, Patarroyo et al. 1987, Herrera et al. 1992). MSP-1 is synthesized by the parasite as a high molecular weight precursor which is cleaved into smaller polypeptide fragments found on the surface of the merozoite (Holder \& Freeman 1984). The gene for MSP- 1 occurs as a dimorphic allele with the amino acids arranged in blocks of conserved and allelespecifc regions (Tanabe et al. 1987). MSP-1 may 
play a role in the merozoite invasion of the red blood cell (Perkins \& Rocco 1988) and antibodies against MSP-1 have been shown to interfere with parasite invasion in vitro (Schmidt-Ulrich et al. 1986, Hui \& Siddiqui 1987, Blackman et al. 1990). B-cell epitopes have been identified in both the $\mathrm{N}$ - and C-terminal regions of the MSP-1 molecule (Sinigalia et al. 1988, Burns et al. 1988, Cooper et al. 1992). Naturally acquired anti-MSP- 1 antibodies have been detected in populations living in malaria endemic regions of the world (Holder \& Freeman 1984, Gabra et al. 1986, Chizzolini et al. 1989, Kramer \& Oberst 1992). Cellular and humoral responses to MSP-1 are acquired in an age-dependent manner and antibodies against specific regions of the MSP-1 molecule have been associated with a decreased frequency of disease (Riley et al. 1992).

In this preliminary cross-sectional study, we examined the malaria parasite prevalence and the humoral immune response to blood stage falciparum malaria antigens in children and adults living in Punta Soldado, Colombia, South America. Specific attention was paid to the detection of naturally acquired antibodies against parasite-derived, recombinant and synthetic MSP-1 antigens of $P$. falciparum.

\section{MATERIALS AND METHODS}

\section{STUDY AREA}

The study site is located in the village of Punta Soldado, a few miles from the pacific coast seaport of Buenaventura in the Department of Valle del Cauca. Malaria in this region is considered unstable. One-hundred and fifty-one individuals, out of a population of approximately 250, agreed to participate in this study. The villagers are of African origin and live in wooden houses along the beach. The main activities of the villagers are farming and fishing. $P$. falciparum is the predominant species followed by $P$. vivax. Severe falciparum malaria is rarely seen in this population (unpublished observation).

\section{SAMPLE COLLECTION}

Demographic data, thick blood smears and peripheral blood for sera were collected from April 5-7, 1993; this time frame corresponds to approximately the beginning of the transmission season (Rojas et al. 1992). The serum samples were stored at $-20^{\circ} \mathrm{C}$ until used. Thick blood smears were stained with
Giemsa stain and examined under oil immersion for the presence of parasites. Parasite density, expressed in parasites $/ \mathrm{mm} 3$, was determined by counting the number of parasites per 100 white blood cells and multiplying the resulting proportion by an average leukocyte count of $8000 / \mathrm{mm}^{3}$. The parasite density was grouped into four classes: class 1: 1 100 , class 2: 101-200, class 3: 201-400, class 4: 401-600 parasites $/ \mathrm{mm}^{3}$.

\section{PARASITE STRAINS AND}

\section{ANTIGENS USED IN THE STUDY}

The FUP (Falciparum-Uganda-Palo Alto) strain of $P$. falciparum was used in this study (Chang et al. 1988). Parasite-derived MSP-1 was isolated by monoclonal antibody affinity chromatography as described previously with the exception that the elution buffer was $0.1 \mathrm{M}$ glycine-HCL, pH 2.5 (Siddiqui et al. 1987). The $\mathrm{N}$-terminal region of MSP-1 (195A) was expressed using a yeast expression system and purified by FPLC as described elsewhere (Hui et al. 1991). 195A is equivalent to the first $56 \%$ of the $83 \mathrm{kDa} \mathrm{N}$-terminal processing fragment. The C-terminal region of MSP-1 (BVp42) was expressed using a baculovirus expression system and purified as described previously (Chang et al. 1992). BVp42 is equivalent to the entire $42 \mathrm{kDa}$ $\mathrm{C}$-terminal processing fragment minus the anchor sequence. The synthetic peptides were derived from predicted $\mathrm{B}$ cell epitopes on the $\mathrm{N}$-terminal region of MSP-1. The sequences of the P. falciparum MSP-1 based synthetic peptides were: peptide 3 (a.a. 203-220; ELLYKLNFYFFDLLRAKLNDVNH2) and peptide 16 (a.a. 333-352; IDTLKKNENIKELLDKINEI-NH2) (amino acid numbering according to Miller et al. 1993). These sequences are found within the FUP sequence. Peptide 3 is within a conserved region (block 3 ) of the molecule while peptide 16 is found within a semiconserved region (block 4).

\section{ANTIBODY DETECTION ASSAYS}

Antibodies against MSP-1 were detected by ELISA. Briefly, antigen $(1 \mu \mathrm{g} / \mathrm{ml}, 50 \mu \mathrm{l} / \mathrm{well})$ diluted in BBS (borate buffered saline; $167 \mathrm{mM}$ boric acid, $134 \mathrm{mM} \mathrm{NaCl}, 27.5 \mathrm{mM} \mathrm{NaOH}, \mathrm{pH} 8.0$ ) was coated to polyvinyl plates for 2 hours in a humid box. Unbound antigen was removed by washing the plate three times with $\mathrm{HSBBS}$ (BBS with $\mathrm{NaCl}$ at $0.5 \mathrm{M}$ ). The wells were blocked with $150 \mu \mathrm{l}$ of $5 \%$ milk in BBS for 2 hours in a humid box. The plate 
was washed three times with HSBBS and frozen at $-70^{\circ} \mathrm{C}$ until needed. The serum samples were diluted $1 / 200$ in BBS with $1.5 \%$ powdered low fat milk. Diluted sera were tested in duplicate wells in which $50 \mu \mathrm{l} /$ well were reacted for 2 hours. The unbound antibodies were removed by washing. Bound antibodies were detected using a goat-anti human IgG peroxidase conjugated antibody (ZYMED, gamma chain specific) diluted in BBS with $1.5 \%$ milk. After two hours the plates were washed and $50 \mu \mathrm{l} /$ well of substrate was added. The OD410 was read after 30 minutes using a Dynatech MR650 Microplate Reader. Antibody units were determined using a standard curve derived from a pool of adult sera from Punta Soldado. Wells were similarly reacted with sera from unexposed persons served as a negative control. An individual was considered negative if the mean OD value of 2 wells was less than the mean plus 2 standard deviations of the negative control wells on each plate.

The IFAT was performed as described elsewhere (Voller 1971). Titers greater than $1 / 16$ were considered positive.

Categorical data were analyzed using the Chi square test. Serological and parasitological data were analyzed using the Mann-Whitney Rank Sum Test for comparison of two groups and the KruskalWallis One Way ANOVA on Ranks to compare multiple groups with Dunn's method to isolate which group(s) were different. Spearman rank order correlation was used to assess the linear relationship between variables. Differences of $p<0.05$ were considered significant.

\section{TABLE}

Parasite and antibody prevalence for Plasmodium falciparum by age group in villagers of Punta Soldado, Colombia

\section{RESULTS}

Fifty $(33.1 \%)$ villagers were positive for $P$. falciparum by Giemsa stained thick blood smears (Table I). During the survey, no severe or complicated cases of falciparum malaria were noted. $P$. vivax parasites were not found. Parasites were not detected in the youngest age group (1-4 years). Infected individuals had parasite densities between 80 and 560 parasites $/ \mathrm{mm}^{3}$. Twenty individuals (40\%) had a class 1 infection, $12(24 \%)$ had a class $2,16(32 \%)$ had a class 3 and $2(4 \%)$ had a class 4 infection. The frequency of parasite positive blood smears increased with age, reaching a maximum of $48 \%$ in the $15-29$ age group. There was no significant difference in the parasite densities between the three age groups (Table I; Kruskal-Wallis One Way ANOVA, $p=0.09$ ).

The overall seroprevalence of anti-P. falciparum antibodies, as measured by IFAT, was $92 \%$. Seventy percent of the youngest age group (1-4years) were IFAT positive (Table I). The IFAT titers of the two older age groups (15-29 and 30+ years) were significantly higher than the IFAT titers of the younger age groups (Kruskal-Wallis One Way ANOVA, $p>0.05$ ). There was no difference in IFAT titers between infected and uninfected individuals (infected individuals: mean $=38$, median $=32$; uninfected individuals: mean $=33$, median $=$ 32; Mann-Whitney Rank Sum Test, p (0.05).

We evaluated the antibody response to the blood stage antigen MSP- 1 by ELISA using purified parasite-derived MSP-1 and the recombinant 
polypeptides 195A and BVp42 (Table II). The correlation coefficients ( $r$ ) between the antibody concentrations against parasite-derived MSP- 1 verses the recombinant antigens were 0.725 and 0.713 for $195 \mathrm{~A}$ and $\mathrm{BVp} 42$ respectively ( $p<0.001$ for both comparisons). The overall seroprevalence for antiMSP-1, anti-195A and anti-BVp42 antibodies was $74 \%, 68 \%$ and $68 \%$ respectively. Both the mean and median antibody units against the three antigens increased with age. In general, the older age groups had higher concentrations of anti-MSP-1 antibodies than did the younger age groups. There was no significant difference between the number of females and males with detectable antibodies to parasite-derived MSP- 1 and $\mathrm{BVp} 42\left(\mathrm{X}^{2}=0.962\right.$ and 2.793 respectively, $p>0.05$ ). However, a larger number of males than females had antibodies which recognized 195A $\left(\mathrm{X}^{2}=5.99, \mathrm{p}<0.05\right)$.

Infection appeared to boost the antibody concentration to MSP-1. Infected individuals had significantly higher anti-MSP- 1 antibody concentrations than non-infected individuals (parasite-derived MSP-1: infected individuals: mean $=224$, median $=26$, non-infected individuals: mean $=125$, median $=7 ; 195 \mathrm{~A}:$ infected individuals: mean $=$ 310 , median $=80$, non-infected individuals: mean $=207$, median $=69 ;$ BVp42: infected individuals: mean $=139$, median $=43$, non-infected individuals: mean $=139$, median $=36 ;$ Mann-Whitney Rank Sum Test, $p=0.02,0.04 \& 0.03$ respectively). Among the infected individuals, boosting of the antibody response was also reflected in a very strong positive correlation between antibody con-

\section{TABLE II}

Anti-MSP-1 antibodies by age group in villagers of Punta Soldado, Colombia

\begin{tabular}{|c|c|c|c|c|c|c|c|c|}
\hline \multirow{4}{*}{ Antigen } & \multirow{4}{*}{$\begin{array}{l}\text { Age Group } \\
\text { (Years) }\end{array}$} & \multicolumn{7}{|c|}{ ELISA results } \\
\hline & & \multirow{3}{*}{$\begin{array}{l}\text { Percent } \\
\text { Antibody } \\
\text { Positive }\end{array}$} & \multirow{3}{*}{$\begin{array}{l}\text { Reciprocal } \\
\text { Mean } \\
\text { Antibody Units }\end{array}$} & \multirow{3}{*}{$\begin{array}{l}\text { Reciprocal Median } \\
\text { Antibody Units }\end{array}$} & \multicolumn{4}{|c|}{ Difference in Rank } \\
\hline & & & & & \multicolumn{4}{|c|}{ Age Group years } \\
\hline & & & & & $1-4$ & $5-14$ & $15-29$ & $30+$ \\
\hline \multirow{5}{*}{ MSA - 1} & $1-4$ & 30 & 3 & 0 & - & - & - & - \\
\hline & $5-14$ & 59 & 69 & 5 & 19.6 & - & - & - \\
\hline & $15-29$ & 86 & 136 & 20 & $46.6^{\star}$ & $27.0^{\star}$ & - & - \\
\hline & $30+$ & 87 & 315 & 59 & $62.2^{\star}$ & $42.6^{*}$ & 15.6 & - \\
\hline & Total & 74 & 159 & 10 & \multicolumn{4}{|c|}{$\begin{array}{l}{ }^{a} \text { Kruskal-Wallis One Way ANOVA, } p<0.001 \\
{ }^{b} \text { Dunn's Comparison, } p<0.05\end{array}$} \\
\hline \multirow{5}{*}{$195 \mathrm{~A}$} & $1-4$ & 40 & 35 & 0 & - & - & - & - \\
\hline & $5-14$ & 55 & 180 & 50 & 14.5 & - & - & - \\
\hline & $15-29$ & 71 & 222 & 73 & 27.4 & 13.0 & - & - \\
\hline & $30+$ & 87 & 397 & 97 & $47.5^{\star}$ & $33.1^{*}$ & 20.1 & - \\
\hline & Total & 68 & 241 & 72 & \multicolumn{4}{|c|}{$\begin{array}{l}{ }^{a} \text { Knuskal-Wallis One Way ANOVA, } \mathrm{p}<0.001 \\
{ }^{b} \text { Dunn's Comparison, } \mathrm{p}<0.05\end{array}$} \\
\hline \multirow{5}{*}{$\mathrm{BVp} 42$} & $1-4$ & 20 & 10 & 0 & - & - & - & - \\
\hline & $5-14$ & 55 & 32 & 33 & 22.9 & - & - & - \\
\hline & $15-29$ & 75 & 109 & 41 & $43.5^{*}$ & 20.6 & - & - \\
\hline & $30+$ & 87 & 184 & 54 & $65.5^{\star}$ & $42.7^{\star}$ & 22.1 & - \\
\hline & Total & 68 & 101 & 39 & \multicolumn{4}{|c|}{$\begin{array}{l}{ }^{a} \text { Kruskal-Wallis One Way ANOVA, } p<0.001 \\
{ }^{b} \text { Dunn's Comparison, } p<0.05\end{array}$} \\
\hline
\end{tabular}


centrations of the three antigens tested $(r=0.998$, $0.994 \& 0.994$ for MSP-1 vs 195A, MSP-1 vs $\mathrm{BVp} 42$ \& 195A vs BVp42 respectively, $\mathrm{p}<0.005$ for all comparisons).

The specificity of the detectable anti-MSP-1 antibodies were analyzed using immunoblotting (data not shown). In general, individuals who recognized the respective antigens by ELISA also recognized appropriate molecular size bands on the immunoblots. Thirty-six sera, which reacted with 83 $\mathrm{kDa} \mathrm{N}$-terminal processing fragment by immunoblotting against parasite-derived MSP-1, were tested by ELISA against two $\mathrm{N}$-terminal synthetic peptides (peptide 3 and peptide 16) derived from conserved 190L region $P$. falciparum MSP-1. P16 has been identified as a MSP-1 / Spectrin binding region (Herrera et al. 1993). Five (13.9\%) of these sera reacted with peptide 3 , eighteen $(50 \%)$ reacted with synthetic peptide 16 . Thirteen sera (36\%) reacted only with peptide 16 . The five individuals who recognized peptide 3 were adults whereas individuals of all age groups, including children, recognized peptide 16 . There was no association between a positive ELISA value for either synthetic peptide and parasite density or MSP-1 (parasitederived MSP-1, 195A and BVp42) antibody concentrations.

\section{DISCUSSION}

In this study, we evaluated the anti- $P$. falciparum antibody response and parasite prevalence and density of 151 asymptomatic individuals from the malaria endemic village of Punta Soldado, Colombia, South America. The overall P. falciparum infection rate was $33.1 \%$. The highest parasite prevalence was found in the 15-29 age group. The maximum prevalence of IFAT anti-falciparum antibodies was not reached until the $30+$ age group. In highly endemic areas of Africa, maximum parasite prevalence is reached in childhood; $\leq 2$ years in holoendemic, 2-4 years in hyperendemic and 5-9 years in mesoendemic regions (Boyd 1949, Schwetz 1949). Under intense malaria transmission, $100 \%$ of the children between 1-4 years would be predicted to be IFAT antibody positive (Voller et al. 1980). In the absence of detailed epidemiological data from Punta Soldado, these two criteria lead us to conclude that transmission in this region is probably low and seasonal. However we can not rule out that the time frame of the study, which was just before the peak transmission season, could have accounted for the low parasite prevalence and densities observed in the study population. On the other hand, the $P$. falciparum infection rates by age group in a placebo group from another Pacific coast village in Colombia (Valero et al. 1993) were very similar to the parasite prevalence data reported in this study. Future studies are planned to accurately define the epidemiology of malaria in Punta Soldado.

Despite significant differences in the antibody response to the blood stage antigens tested, the similar low parasite densities noted in the three age groups suggests that this population's resistance to severe falciparum infections may be the result of several mechanisms rather than solely due to acquired immunity. Other reasons for this observation may be genetic factors such as the presence in this population of haemoglobin $\mathrm{S}$, thalassemias and glucose-6-phosphate dehydrogenase deficiency or the unreported use of antimalarial drugs or folk medicines (Bruce-Chwatt 1990).

When parasite-derived MSP-1 was used in an ELISA assay, $74 \%$ of the people of Punta Soldado had detectable antibodies to this prominent merozoite surface polypeptide. Both prevalence and concentration of anti-MSP-1 antibodies increased with age; indicating a positive relationship between exposure to the parasite and the development of anti-MSP-1 antibodies. Furthermore, infected individuals had higher concentrations of anti-MSP-1 antibodies than non-infected individuals. The prevalence of anti-MSP-1 antibodies in a population would be expected to indicate the intensity of transmission for a given edemic area. In the Gambia, Gabra et al. (1986), using an $E$. coli recombinant polypeptide (31-1) representing a portion of the $83 \mathrm{kDa} \mathrm{N}$-terminal processing fragment, reported a high proportion $(>90 \%)$ of individuals, 5 years and older, with positive ELISA values. Kramer and Oberst (1992), also, found a seroprevalence of $90 \%$ of anti-MSP-1 antibodies in a population living in a hyperendemic area of the Philippines. Conversely, using the 31-1 antigen, Chizzolini et al. (1989) reported a much lower prevalence $(\sim 15 \%)$ of anti-MSP-1 antibodies in a population living in a low malaria transmission region of Gabon. The similar seroprevalence rates and high correlation of antibody concentrations for the three antigens tested in this study, which included a purified parasite-derived polypeptide (MSP- 
1) and two recombinant polypeptides (195A and BVp42), suggests a large overlap of epitopes present on the parasite and recombinant polypeptides.

The humoral responses to the two recombinant antigens, $195 \mathrm{~A}$ and $\mathrm{BVp} 42$, were very similar to the parasite-derived MSP-1 antigen . According to our data, the prevalence and concentration of antibodies to MSP-1 increased with age with equal immunogenicity to both $\mathrm{N}$-terminal and $\mathrm{C}$-terminal regions of the molecule. In fact, the antibody prevalence and concentration for the three MSP-1 antigens continued to increase with age. The recombinant antigens used in this study were based on the reported sequence of the FUP strain of $P$. falciparum. The representation of this dimorphic allele within the parasite population of Punta Soldado needs to be investigated. The synthetic peptides used in this study were derived from predicted $B$ cell epitopes within the $83 \mathrm{kDa} \mathrm{N}$-terminal processing fragment. Fifty percent of the tested sera reacted with one of the two synthetic peptides, which included a semi-conserved site, indicates that these B cell epitopes are present in the parasite population of Punta Soldado. However we were unable to find an association between individuals which reacted with the peptides and parasite density or antibody concentrations to MSP-1. This may be due to the small number of serum samples tested and an expanded study using these peptides is planned.

Studies are presently underu ay to determine the IgG subclass of anti-MSP-1 antibodies and to examine the in-vitro growth inhibition of the serum samples collected in this study. Future studies are planned in Punta Soldado to define the epidemiology of $P$. falciparum as well as to examine the relative contributions of immune and nonimmune factors in resistance to severe falciparum malaria in this population.

\section{ACKNOWLEDGEMENT}

To the residents of Punta Soldado for their participation in these studies, to Drs Maria Fernanda Rizzo and Nancy Torres for field and technical assistance.

\section{REFERENCES}

Blackman MJ, Heidrich HG, Donachie S, McBride J, Holder A 1990. A single fragment of a malaria merozoite surface protein remains on the parasite during red cell invasion and is the target of invasion-inhibiting antibodies. J Exp Med 172: 379-382.

Boyd M 1949. Epidemiology of malaria: factors related to the intermediate host. In Malariology. Saunders Company (Philadelphia), p. 551-607.

Bruce-Chwatt L 1980. In Essential Malariology. William Heinemann Medical Books Ltd. (London). p. 58-59.

Burns J, Daly T, Vaidya A, Long C 1988. The 3' portion of the gene for Plasmodium yoelii merozoite surface antigen encodes the epitope recognized by protective monoclonal antibody. Proc Natl Acad Sci USA 85: 602-606.

Chang S, Gibson H., Lee-Ng C, Barr P, Hui G 1992. A carboxil-terminal fragment of Plasmodium falciparum gp195 expressed by a recombinant baculovirus induces antibodies that completely inhibit parasite growth. J Immunol 149: 548-555.

Chang S, Kramer K, Yamaga K, Kato A, Case S, Siddiqui W 1988. Plasmodium falciparum: gene structure and hydropathy profile of the major merozoite surface antigen (gp 195) of the Uganda-Palo Alto isolate. Exp Parasitol 67: 1-11.

Cheung A, Leban J, Shaw A 1986. Immunization with synthetic peptides of Plasmodium falciparum surface antigen induces antimerozoite antibodies. Proc Natl Acad Sci USA 83: 8328-8332

Chizzolini C, Delaporte E, Kaufmann J, Akue J, Verdini A, Pessi A, del Giudice G 1989. Age-related prevalence of antibody response against three different, defined Plasmodium falciparum antigens in children from the Haut-Ogooue Province in Gabon. Trans Roy Soc Trop Med Hyg 83: 147-151.

Cooper J, Cooper L, Saul A 1992. Mapping of the region predominantly recognized by antibodies to the Plasmodium falciparum merozoite surface antigen MSA1. Molec Biochem Parasit 51: 301-312.

Gabra J, Grossiord D, Perrin L, Shaw A, Cheung A, MacGregor I 1986. Defined Plasmodium falciparum antigen in malaria serology. Bull WHO 64: 889-896.

Hall R., Hyde J, Goman M, Simmons D, Hope I, Mackay, J, Scaife J, Merkli B, Richie R., Stocker J 1984. Major surface antigen gene of a human malaria parasite cloned and expressed in bacteria. Nature 311 : 379-382.

Herrera MA, Rosero F, Herrera S, Caspers P, Rotman D, Sinigaglia F, Certa U 1992. Protection against malaria in Aoutus monkeys immunized with a recombinant blood stage antigen fused to a universal $T$ cell epitope: correlation of serum gamma interferon levels wiyh protection. Infect Immun 60: 154-158

Holder A, Freeman R 1984. The three major antigens on the surface of Plasmodium falciparum merozoites are 
derived from a single high molecular weight precursor. J Exp Med 160:624-629.

Hui G, Chang S, Gibson H, Hashimoto A, Hashiro C, Barr $P$, Kotani S. 1991. Influence of adjuvants on the antibody specificity to the Plasmodium falciparum major merozoite surface protein, gp 195. J Immunol 147: 3995-3941.

Hui G, Siddiqui W 1987. Serum from Pf 195 protected Aotus monkeys inhibit Plasmodium falciparum growth in vitro. Exp Parasitol 64:519-522.

Kramer K., Oberst R 1992. Antibodies to the major merozoite surface coat protein of Plasmodium falciparum (gp195) in a human population living in a malaria endemic area of the Philippines. Am J Trop Med Hyg 47: 429-439.

McGregor IA, Wilson, R JM 1988. Specific immunity: acquired in man. In Malaria. Principles and Practice of Malariology. Vol. I. Ed. W. H. Wernsdorfer \& I. McGregor. Churchill Livingstone. (EdinburghLondon-Melboume-New York). p. 559-619.

Miller L, Roberts T, Shahabuddin M, McCutchan T 1993. Analysis of sequence diversity in the Plasmodium falciparum merozoite surface protein-1 (MSP -1). Mol Biochem Parasitol 59: 1-14.

Patarroyo M, Romero P. Torres M, Clavijo P, Moreno A, Martinez A, Rodríguez R, Guzmán F, Cabezas E 1987. Induction of protective immunity aginst experimental infection with malaria using synthetic peptides. Nature 328: 629-632.

Perkins M, Rocco I 1988. Sialic acid dependent binding of Plasmodium falciparum merozoite surface antigen, Pf200, to human erythrocytes. J Immunol 141: 3190-3196.

Perrin I, Merkli B, Loche M, Chizzolini C, Smart J, Richle R. 1984. Antimalarial immunity in Saimiri monkeys. Immunization with surface components of asexual blood stages. J Exp Med 160: 441-451.

Riley E, Allen S, Wheeler J, Blackman M, Bennett S, Takacs B, Schonfeld H, Holder A, Greenwood B 1992. Naturally acquired cellular and humoral immune responses to the major merozite surface antigen (PfMSP-1) of Plasmodium falciparum are associated with reduced malaria morbidity. Parasite Immunol 14: 321-337.

Rojas W, Penaranda F, Echavartia M 1992. Strategies for malaria control in Colombia. Parasit Today 8: 141144.
Schmidt-Ulrich R, Brown J, Whittle H, Peck-Sun L, 1986. Human-hybridomas secreting monoclonal antibodies to the $\mathrm{Mr} 195,000$ Plasmodium falciparum blood stage antigen. $J$ Exp Med 163: 179-188.

Schwetz J 1949. Notes on endemic and acute malaria in central African natives. Trans Roy Soc Trop Med Hyg 42: 403-408.

Siddiqui W, Tam L, Kramer K, Hui G, Case S, Yamaga K, Chang S, Chan E, Kan S 1987. Merozoite surface coat precursor protein completely protects Aotus monkeys against Plasmodium falciparum malaria. Proc Natl Acad Sci USA 84: 314-318.

Sinigaglia F, Takacs B, Jacot H, Matile H, Pink J, Crisanti A, Bujard H. 1988. Nonpolymorphic regions of p190, a protein of the Plasmodium falciparum erythrocytic stage, contain both $\mathrm{T}$ and $\mathrm{B}$ cell epitopes. $J$ Immunol 140: 3568-3572.

Tanabe K, Mackay M, Goman M, Scaife J 1987. Allelic dimorphism in a surface antigen gene of the malaria parasite Plasmodium falciparum. J Mol Biol 195: 273-287.

Taylor DW 1989. Humoral immune responses in mice and man to malarial parasites. In Malaria: Host Responses to Infection. Ed. M. Stevenson. CRC Press, Inc. (Boca Raton).

Tolle R, Fruh K, Doumbo O, Koita O, NDiaye M, Fischer A, Dietz K, Bujard H 1993. A prospective study of the association between the human humoral immune response to Plasmodium falciparum blood stage antigen gp190 and control malarial infections. Infect. Immunity 61(1):40-47.

Valero M., Amador L, Galindo C, Figueroa J, Bello M, Murillo L, Mora A, Patarroyo G, Rocha C, Rojas M, Aponte J, Sarmiento L, Lozada D, Coronell C, Ortega N, Rosas J, Alonso P, Patarroyo M 1993. Vaccination with SPf66, a chemically synthesized vaccine, against Plasmodium falciparum malaria in Colombia. Lancet 341: $705-710$.

Voller A 1971. The detection and measurement of malaria antibodies. Trans R Soc Trop Med Hyg 65: 111-124.

Voller A, Cornille-Brogger R, Storey J, Molineaux L 1980. A longitudinal study of Plasmodium falciparum malaria in the West African savanna using the ELISA technique. Bull WHO 58: 429-438. 